\title{
Ten-year survival after portal vein arterialization in liver transplantation
}

\author{
Yuan Cheng ${ }^{1,2}$, Yongbiao Chen ${ }^{2}$, Yi Jiang ${ }^{2}$, Xiaojin Zhang ${ }^{2}$ \\ ${ }^{1}$ Graduate School, Fujian Medical University, Fuzhou 350004, China; ${ }^{2}$ Department of Hepatobiliary Surgery, 900 Hospital of the Joint Logistics \\ Team, PLA, Fuzhou 350025, China \\ Correspondence to: Xiaojin Zhang. Department of Hepatobiliary Surgery, 900 Hospital of the Joint Logistics Team, PLA, No. 156, West Second Ring \\ Road North, Gulou District, Fuzhou 350025, China. Email: DrZhXJ@163.com.
}

Submitted Sep 09, 2019. Accepted for publication Oct 21, 2019.

doi: 10.21037/apm.2019.11.01

View this article at: http://dx.doi.org/10.21037/apm.2019.11.01

Portal vein thrombosis (PVT) remains a technical difficulty for orthotopic liver transplantation (OLT) due to the need of complex vascular reconstruction to maintain portal blood flow into the allograft (1). Direct arterialization of the donor portal vein $(\mathrm{PV})$ is a relatively simple method of improving the portal inflow, though there is little information regarding long-term results after this procedure (2-4).

Our center previously reported two patients who underwent portal vein arterialization (PVA) for preexisting thrombosis during OLT (5). A 3-year uneventful postoperative course of the first patient was reported in that paper, though the second patient died of severe pulmonary infection and acute renal failure on the $60^{\text {th }}$ postoperative day (POD). More than 10 years after the surgery, the first patient is still doing well with normal graft function. To our knowledge, this is the first case of more than 10-year survival after permanent PVA in a liver transplant patient. Accordingly, we discuss in detail our experience with this surgical technique.

A 50-year-old man, who was diagnosed with hepatitis B virus (HBV)-related decompensated liver cirrhosis, complicated with portal hypertension, PVT, hypersplenism, ascites, and hemorrhage of the upper alimentary tract, was placed on the waiting list for OLT. Two months after admission, this patient underwent simultaneous splenectomy plus OLT on October 15, 2008.

Intraoperatively, hepatic lobar atrophy, numerous venous collaterals in the duodenal and pancreatic areas, splanchnic vasodilation and splenomegaly were found. Besides, intraoperative ultrasound test indicated diffuse thrombosis in the PV with invasion into the superior mesenteric vein.
In an attempt to recanalize the PV, we performed a direct portal eversion and thrombectomy, followed by an endto-end portal venous anastomosis. Unfortunately, the portal blood flow did not resume after portal unclamping due to renewed venous thrombosis. Hence, the portal anastomosis was removed and the recipient PV stump was ligated. In consideration of severe coagulopathy caused by decompensated hepatic cirrhosis, it was at great risk of massive hemorrhage to try bypass or hemitransposition. Eventually, we decided to perform PVA using a readily attainable recipient middle colic artery (MCA). We first isolated and transected the recipient MCA, created a trumpet-shaped terminal, and then anastomosed this artery to the donor PV stump with running sutures in an end-toend fashion. Afterwards, arterial inflow of the allograft was reconstructed by an end-to-end anastomosis of the common hepatic artery. Finally, another ultrasound test showed that intrahepatic portal and arterial blood flow were good, though the exact details of pressure and velocity were not recorded.

Postoperatively, the patient received tacrolimus and steroid immunosuppression therapy and betablocker treatment with propranolol at a dosage of $300 \mathrm{mg} / \mathrm{d}$. He made uneventful postoperative recovery, without significant ascites or hematemesis. Ultrasound examination performed on the fourth POD revealed adequate perfusion of the allograft liver and good arterialized blood flow permeating through the graft portal system [peak systolic velocity (PSV): approximately $0.19-0.29 \mathrm{~m} / \mathrm{s}]$. The blood vessel diameters (BVDs) of the PV in the hepatic hilus region, the anastomotic stoma of the PVA and its distal MCA were 

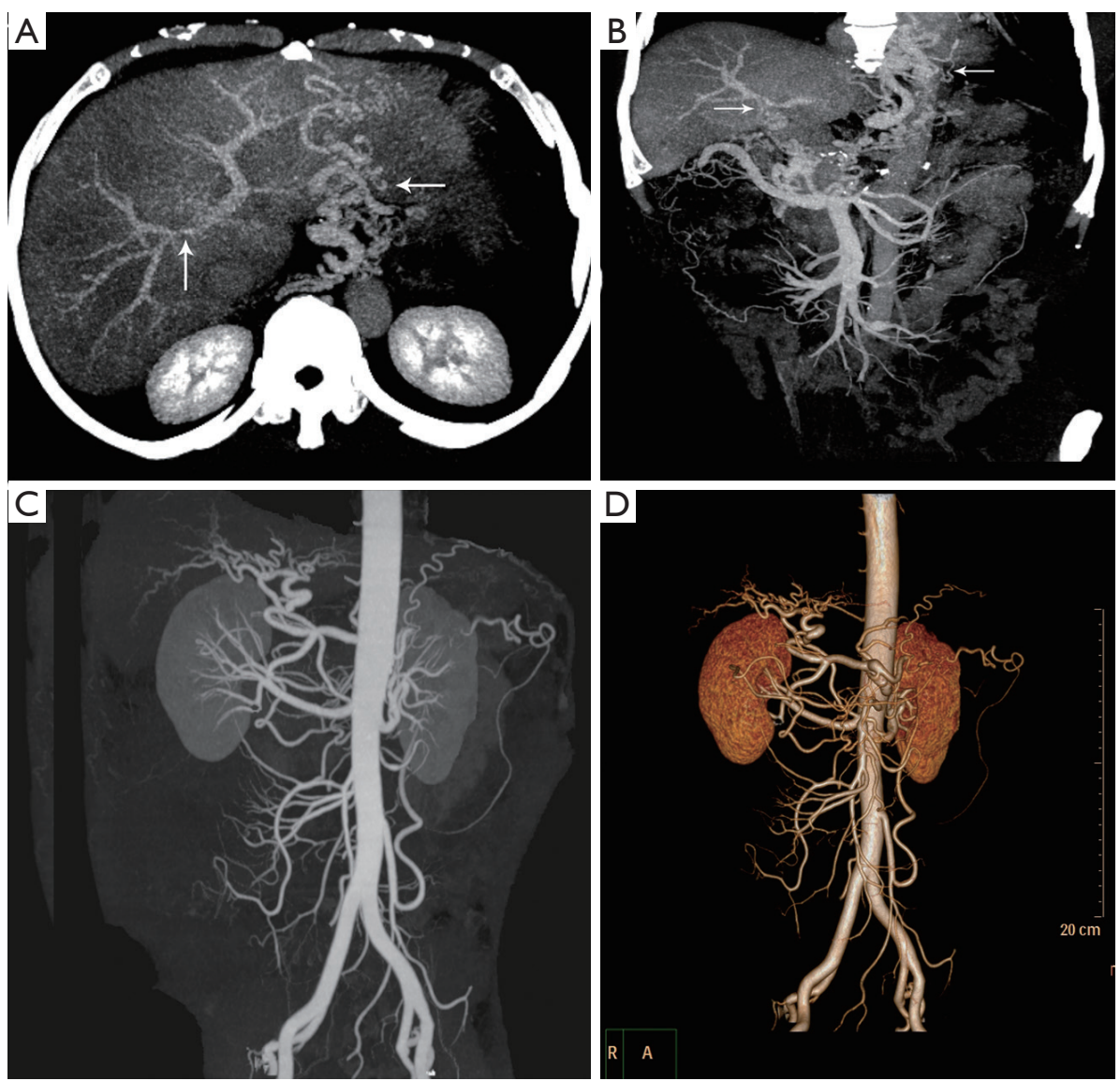

Figure 1 Abdominal CTA examination. (A) Horizontal scanning image at the portal venous phase processed via the MIP technique, mild poor visualization of the right branch of PV (vertical arrow), numerous vasodilated splanchnic collaterals (horizontal arrow); (B) coronal scanning image at the portal venous phase processed via the MIP technique, mild poor visualization of the main trunk of PV (rightward arrow), numerous vasodilated splanchnic collaterals (leftward arrow); (C) image at the hepatic arterial phase processed via the MIP technique; (D) three-dimensional image of the celiac artery. CTA, computed tomography angiography; MIP, maximal intensity projection; PV, portal vein.

1.0, 0.68 and $0.92 \mathrm{~cm}$, respectively. The PSV and resistance index (RI) of the MCA were $1.71 \mathrm{~m} / \mathrm{s}$ and 0.41 , respectively. The intrahepatic arterial PSV, BVD and RI were $0.81 \mathrm{~m} / \mathrm{s}$, $0.40 \mathrm{~cm}$ and 0.73 , respectively.

Three years after surgery, abdominal computed tomography angiography (CTA) showed a slightly poor visualization of the main trunk and right branch of the PV and numerous vasodilated splanchnic collaterals in the periphery of the esophagus-cardia-fundus ventriculi (Figure $1 A, B)$; mild intra- and extrahepatic arterial dilatation were also found (Figure 1C,D). CTA performed 5 and 7 years after OLT showed no changes compared to the previous tests. Furthermore, no significant organic disease inside the upper alimentary tract was detected by a barium swallow test performed in the third postoperative year. More than ten years after the surgery, this patient remains healthy without any complication, and fluctuation in the allograft function has seldom been observed.

Existing data on the effects of permanent PVA demonstrate that the major complications of this technique are related to "overarterialization". Hyperperfusion of the portal inflow into the liver graft may lead to hepatic fibrosis and right heart failure (2). Furthermore, Bonnet et al. (3) reported a case of PVA during OLT with the development of a diffuse aneurismal dilatation of the intrahepatic portal venous branches during a 6-year follow-up period. In some clinical series $(3,6,7)$ and experimental investigations (8), a technique for limiting the blood volume in the arterialized 
PV has been performed to prevent the progress of liver fibrosis and right heart decompensation. However, there is no uniform and definite view of the optimal volume for the arterialized portal flow. Paloyo et al. (4) presented a patient who underwent PVA during OLT with the usage of an accessory right hepatic artery without calibration of the arterioportal anastomosis, and no proof of right heart overload, biliary stricture, or stenosis was found within a 4-year follow-up period.

In the present patient, we did not deliberately calibrate the arterioportal anastomosis. In the ten years after surgery, CTA examinations showed mildly poor visualization of the PV and its intrahepatic branches, which might be the result of luminal stenosis of arterioportal anastomosis caused by running sutures. Simultaneously, the hepatic arterial system compensatorily dilatated to maintain sufficient blood flow into the liver graft. As far as the results are concerned, a long-term survival with normal graft function confirms that the entire hepatic blood supply is completely adequate. Nevertheless, the technique of PVA is an unphysiological vascular reconstruction and does not address the portal hypertension. Apparently, splenectomy and persistent betablocker treatment effectively limited the splanchnic blood pressure, and no hemorrhage of the upper alimentary tract recurred.

In summary, PVA is a technically simpler option for non-recanalizable portal systematic thrombosis within the context of OLT. To date, there have only been anecdotal reports on the long-term results of this technique. Our experience demonstrates a favorable outcome of permanent PVA with the use of the recipient MCA.

\section{Acknowledgments}

We thank Dr. He Tong and Dr. Kejie Yin (Medical Image Center, Fuzhou General Hospital) for technical support in processing the computed tomography examination images. Funding: This work was supported by the Qihang Fund of Fujian Medical University (2018QH2042); and the Natural Science Fund of Fujian Province, China (2018J01351).

Cite this article as: Cheng Y, Chen Y, Jiang Y, Zhang X. Ten-year survival after portal vein arterialization in liver transplantation. Ann Palliat Med 2019;8(5):790-792. doi: 10.21037/apm.2019.11.01

\section{Footnote}

Conflicts of Interest: The authors have no conflicts of interest to declare.

Ethical Statement: The authors are accountable for all aspects of the work in ensuring that questions related to the accuracy or integrity of any part of the work are appropriately investigated and resolved.

\section{References}

1. Lladó L, Fabregat J, Castellote J, et al. Management of portal vein thrombosis in liver transplantation: influence on morbidity and mortality. Clin Transplant 2007;21:716-21.

2. Ott R, Böhner C, Müller S, et al. Outcome of patients with pre-existing portal vein thrombosis undergoing arterialization of the portal vein during liver transplantation. Transpl Int 2003;16:15-20.

3. Bonnet S, Sauvanet A, Bruno O, et al. Long-term survival after portal vein arterialization for portal vein thrombosis in orthotopic liver transplantation. Gastroenterol Clin Biol 2010;34:23-8.

4. Paloyo S, Nishida S, Fan J, et al. Portal vein arterialization using an accessory right hepatic artery in liver transplantation. Liver Transpl 2013;19:773-5.

5. Zhang K, Jiang Y, Lv LZ, et al. Portal vein arterialization technique for liver transplantation patients. World J Gastroenterol 2014;20:12359-62.

6. Stange B, Glanemann M, Nüssler NC, et al. Indication, technique, and outcome of portal vein arterialization in orthotopic liver transplantation. Transplant Proc 2001;33:1414-5.

7. Settmacher U, Stange B, Schaser KD, et al. Primary permanent arterialization of the portal vein in liver transplantation. Transpl Int 2003;16:430-3.

8. Müller V, Brummer D, Kissler H, et al. Effects of portal vein arterialization on regeneration and morphology in liver transplantation: investigations using the rat model. Transplantation 2004;78:1159-65. 